\title{
A Study on Location-Route Optimization Model of Logistics Distribution Center and Its Heuristics Solving Algorithm in Multi- Modal Transport Network
}

\author{
Hui HU ${ }^{1}$, Jianliang LI, and Xudong ZHAO \\ Economics and Management School, East China Jiaotong University, PRC, 330013
}

\begin{abstract}
Taking environmental concerns into consideration, a logistics distribution center location-route multi-objective optimization model and its solving algorithm are studied in multi-modal transport network context. The objective functions in the model include total operation cost, delivery time and carbon emission goals. The model's decision variables are product volumes with different transport modes and the constraints concerned with investment budget, limited capacity etc. Aimed at the model structure, a two-stage heuristic solving algorithm for single objective model is put forward and its validity is proved. On the basis of solutions which are searched by the heuristic solving algorithm, an optimal solution is obtained using one of multi-objective evaluation methods. Finally, a large scale multi-modal distribution network example is provided to illustrate feasibility and effectiveness of the model and the algorithm by comparing solving efficiency and results, and it finds a railway-based multi-modal transport network has the most competitive advantage.
\end{abstract}

Keywords. Multi-modal transport, location-route problem, heuristic algorithm, green logistics

\section{Introduction}

As an important role in logistics and supply chain, transportation not only exerts various impacts on supply chain such as delivery, logistic cost, inventory management etc. [1], but also produces undesirable effects on environment and society, for instance, exhausts emission, noise pollution and traffic safety [2] etc. According to statistics investigation, environmental pollution caused by freight accounts for $14 \%$ among the total pollutions, and the percentage of transportation cost in the whole logistics cost is $30-45[3,4]$. There are multiple transport modes such as road, railway, water and airline, which have their own advantages and disadvantages, and it will face difficulty in meeting requirements, for example, quick response to market, low logistics cost and sustainable supply chain for a single transport mode. Consequently, a reasonable configuration of multiple transport modes, namely multi-modal transport, is becoming a realistic selection for goods distribution.

${ }^{1}$ Hui HU, associate professor of East China Jiaotong University, Shuangang East Street 808, Nanchang City, Jiangxi Province, PRC; E-mail: hh24895@163.com. 
Along with the transformation from traditional single transport mode to multimodal transport mode in modern logistics, researches on multi-modal transport network models and their solving algorithms is gaining more and more attention. Bauer et al (2009) proposed an integer program in the form of a linear cost, multi commodity, capacitated network design formulation that minimizes the amount of greenhouse gas emissions of transportation activities [5]. Anthony Beresford et al (2011) analyzed available multimodal transport route variations for iron ore shipments from northwest Australia to northeast China, focusing on a major iron and steel manufacturer, and the most competitive multimodal transport route, at least in the short to medium term, is found to be a rail-sea-road combination via Port Bayuquan in China [6]. J.H. Cho et al (2012) presented a weighted constrained shortest path problem (WCSPP) model and a dynamic programming algorithm to draw optimal intermodal freight routing with regard to international logistics of container cargo for export and import [7]. Arnab Bhattacharya (2013) developed an approach for intermodal network design based on traffic flow prediction and network optimization via dynamic reallocation/reconfiguration between road and rail networks [8]. Marco Bortolin et al (2016) put forward a three-objective distribution planner to tackle the tactical optimization of fresh food distribution networks considering operating cost, carbon footprint and delivery time goals [9]. Y. Zhao et al (2018) introduced a stochastic intermodal service network design problem in a sea-rail transportation system, which considers stochastic travel time, stochastic transfer time, and stochastic container demand, a hybrid heuristic algorithm incorporating sample average approximation and ant colony optimization is employed to solve this model [10]. Jiehui Jiang et al (2019) investigated a multimodal green logistics network design problem of urban agglomeration with stochastic demand, in which different logistics authorities among the different cities jointly optimize the logistics node configurations and uniform carbon taxes over logistics transport modes to maximize the total social welfare of urban agglomeration and consider logistics users' choice behaviors [11].

In this paper, taking capacity of distribution center as variable, a multi-objective optimization model on location and route of logistics distribution center in multi-modal transport network is introduced and the solving algorithm is designed using a two-stage heuristic algorithm. The main contribution of this work lies in that it will optimize the location selection and transportation route under investment constraint. By introducing a large scale numerical example, the model's feasibility and the solving algorithm' efficiency are validated.

\section{Model Proposed}

\subsection{Problem Description}

We study the transportation network model in Figure.1, which shows the logistics distribution network diagram via multi-modal transport. After various goods are produced in supply districts, they're transferred from delivery places to distribution centers, then arriving in receipt places until to their final demand destinations. The problem can be depicted as following: subjected to the total investment, what distribution centers should be selected, what is their capacity, and what kind of transport mode the goods routes should choose so that the total operation costs 
including production costs, distribution center construction costs and transportation costs) are minimized in minimal delivery time and least emissions during transportation.

For convenience of modelling, assumptions are given as followed.

- Supply points of goods are delivery places, demand points of goods are goods destinations.

- Goods must be transferred from distribution centers without direct shipment from delivery points to destinations directly and no goods transfers among distribution centers.

- Construction and operation costs of distribution center are monotonically increasing with a linear relationship concerned with the handling capacity, i.e., the more capacity of distribution center, the more costs.

- Economy of scope in the transfer point is embodied owing to efficient collaboration among multiple transport modes, that's to say, transportation cost can be reduced, which may be decided by coefficient of scope economy.

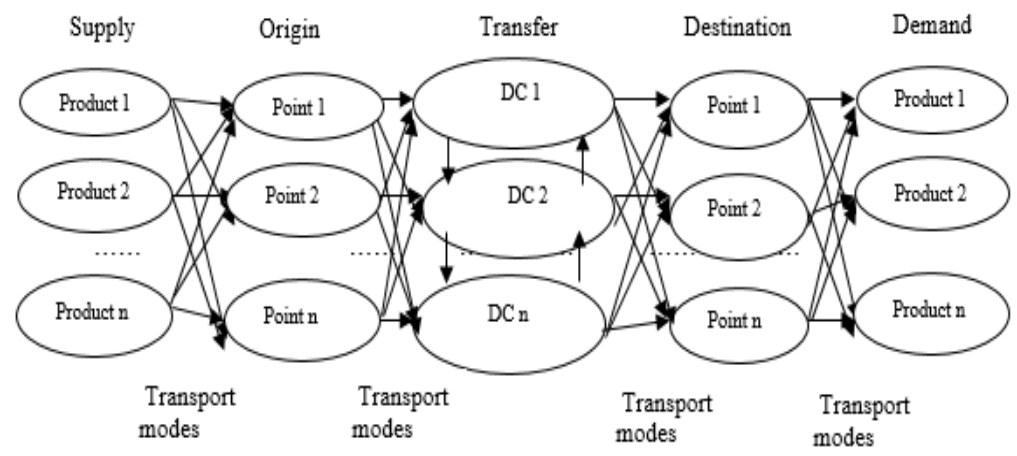

Figure 1. Goods distribution network in multi-modal transport context.

\subsection{Model Symbols}

The sets, parameters, variables in the model are depicted in Table 1, Table 2.

Table 1. Sets in the model

\begin{tabular}{lc}
\hline \multicolumn{1}{c}{ Sets } & Symbol \\
\hline \multicolumn{1}{c}{ Products } & $\mathrm{I}, i \in I$ \\
Origin delivery points & $\mathrm{M}, \boldsymbol{m} \in \boldsymbol{M}$ \\
Distribution center transfer points & $\mathrm{H}, h \in H$ \\
Destination points & $\mathrm{N}, \boldsymbol{n} \in N$ \\
Transport modes & $\mathrm{K}, \boldsymbol{k} \in K$ \\
Transportation path & $\mathrm{P}, p \in P$ \\
\multicolumn{1}{c}{ Emissions } & $\mathrm{E}, e \in E$ \\
\hline
\end{tabular}


Table 2. Parameters, variables in the model

\begin{tabular}{|c|c|c|}
\hline Symbol & $\begin{array}{l}\text { Parameter } \\
\text { Variable }\end{array}$ & Description \\
\hline$a m_{i m p}$ & parameter & $\begin{array}{l}\text { If the origin delivery point of product } \mathrm{i} \text { in the path } \mathrm{m} \text {, it's set } 1 \text {, } \\
\text { other } 0 .\end{array}$ \\
\hline$a n_{i n p}$ & parameter & $\begin{array}{l}\text { If the demand destination point of product } \mathrm{i} \text { in the path } \mathrm{m} \text {, it's set } \\
\qquad 1 \text {, other } 0\end{array}$ \\
\hline cap $_{\text {im }}$ & parameter & The output of product $\mathrm{i}$ in supply point $\mathrm{m}$ \\
\hline$C S_{i h}$ & parameter & Unit storage cost of product $i$ in distribution center $h$ \\
\hline$c t_{i p}$ & parameter & Unit transportation cost of product $\mathrm{i}$ in path $\mathrm{p}$ \\
\hline dem $_{\text {in }}$ & parameter & Demand quantity of product $\mathrm{i}$ in destination point $\mathrm{n}$ \\
\hline$d_{m h}^{k}$ & parameter & $\begin{array}{l}\text { Distance between origin point } \mathrm{m} \text { and distribution center } \mathrm{h} \text { via } \\
\text { transport mode } \mathrm{k}\end{array}$ \\
\hline$d_{h n}^{k}$ & parameter & $\begin{array}{l}\text { Distance between distribution center } \mathrm{h} \text { and destination } \mathrm{n} \text { via } \\
\text { transport mode } \mathrm{k}\end{array}$ \\
\hline$e S_{i h}^{e}$ & parameter & $\begin{array}{l}\text { Unit emission cost of emission e in distribution center } \mathrm{h} \text { storing } \\
\text { product } \mathrm{i}\end{array}$ \\
\hline$e t_{i p}^{e}$ & parameter & Unit emission cost of emission e produced by product $i$ in path $p$ \\
\hline$S_{h}$ & parameter & Scope economy coefficient of distribution center $h$ \\
\hline$t s_{i h}$ & parameter & Storage time of product $\mathrm{i}$ in distribution center $\mathrm{h}$ \\
\hline$t t_{i p}$ & parameter & Transportation time of product $i$ in path $p$ \\
\hline$\alpha^{k}\left(d^{k}\right)$ & parameter & $\begin{array}{l}\text { Unit transportation cost function of transport mode } \mathrm{k} \text {, related with } \\
\text { distance }\end{array}$ \\
\hline$\beta^{k}\left(d^{k}\right)$ & parameter & $\begin{array}{l}\text { Transportation time function of transport mode k, related with } \\
\text { distance }\end{array}$ \\
\hline$\gamma_{k}^{e}\left(d^{k}\right)$ & parameter & Unit emission function of transport mode $\mathrm{k}$, related with distance \\
\hline $\operatorname{In} v^{h}\left(y_{h}\right)$ & parameter & $\begin{array}{l}\text { Construction cost function of distribution center } h \text {, related with } \\
\text { capacity }\end{array}$ \\
\hline$B$ & parameter & Total construction budget of distribution center \\
\hline$\rho$ & parameter & Weighted coefficient in the distribution center total operation cost \\
\hline$L B_{h}, U B_{h}$ & Parameter & Lower limit, upper limit of distribution center's capacity \\
\hline$x_{h}$ & variable & If distribution center $\mathrm{h}$ is chosen, 1 , else, 0 \\
\hline$y_{h}$ & variable & Capacity of distribution center $\mathrm{h}$ \\
\hline$z_{i p}$ & variable & $\begin{array}{l}\text { Volume of product } \mathrm{i} \text { via transportation path } \mathrm{p} \text { from origin delivery } \\
\text { point } \mathrm{m}\end{array}$ \\
\hline
\end{tabular}

\subsection{Objectives and Constraints}

Three objective functions and constraints in the model are expressed in Eq. (1)-(13).

$$
\begin{aligned}
& \text { Obj_Cost }=\rho \frac{\sum_{p} \sum_{i} c t_{i p} \cdot z_{i p}}{\sum_{n} \sum_{i} d e m_{i n}}+\frac{\sum_{h} x_{h} \cdot \operatorname{Inv}^{h}\left(y_{h}\right)}{\sum_{n} \sum_{i} \operatorname{dem}_{i n}} \\
& \text { Obj_Time }=\frac{\sum_{p} \sum_{i} t t_{i p} \cdot z_{i p}}{\sum_{n} \sum_{i} \operatorname{dem}_{i n}}
\end{aligned}
$$


$O b j_{-}$Emission $=\frac{\sum_{p} \sum_{i} \sum_{e} e t_{i p}^{e} \cdot z_{i p}}{\sum_{n} \sum_{i} \operatorname{dem}_{i n}}$

In Eq. (1), total operation cost is computed by adding transportation cost, construction cost of distribution center. Total delivery time is calculated in Eq. (2). Eq. (3) computes total emissions.

$$
\begin{aligned}
& \sum_{p} \operatorname{am}_{i m p} . z_{i p} \leq c a p_{i m} \quad \forall i, m \\
& \sum_{p} \mathrm{a} n_{i n p} \cdot z_{i p} \geq \operatorname{dem}_{i n} \quad \forall i, \mathrm{n} \\
& c t_{i p}=\alpha^{k}\left(d_{m h}^{k}\right)+(1-\mathrm{s}) \alpha^{k}\left(d_{h n}^{k}\right)+c \mathrm{~s}_{i h} \quad \forall i, p \\
& t t_{i p}=\beta^{k}\left(d_{m h}^{k}\right)+\beta^{k}\left(d_{h n}^{k}\right)+\mathrm{ts}_{i h} \quad \forall i, p \\
& e t_{i p}^{e}=\gamma_{k}^{e}\left(d_{m h}^{k}\right)+(1-\mathrm{s}) \gamma_{k}^{e}\left(d_{h n}^{k}\right)+e s_{i h}^{e} \quad \forall i, p, e \\
& \sum_{h} x_{h} \cdot \operatorname{In} v^{h}\left(y_{h}\right) \leq B \\
& \sum_{p} \sum_{i} z_{i p} \leq y_{h} \quad \forall h \\
& x_{h} \in\{0,1\} \quad \forall h \\
& L B_{h} \leq y_{h} \leq U B_{h} \quad \forall h \quad \text { If } x_{h}=1 \\
& z_{i p} \geq 0 \quad \forall i, p
\end{aligned}
$$

In constraint (4), (5), for a given product, supplier, total volumes of transported products in all paths cant' exceed the product capacity and should meet the product demands. Unit transportation cost, time and emission are calculated in Eq. (6), (7), and (8) for a given product, path, emission. Amount of investment is decided by Eq. (9), which means it is allowed within total budget. Freight handling capacity of a given distribution center is restricted as in Eq. (10). The variables' values are determined by Eq. (11), (12) and (13). The distribution center selection is a binary variable and its capacity is limited to an interval, while volumes transported in a path should be positive. 


\section{Two-stage Heuristic Algorithm for the Model}

\subsection{Two-stage Heuristic Algorithm}

A two-stage heuristic algorithm is developed to solve the proposed model. On one hand, this multi-objective optimization model can firstly be solved to minimize one of objective functions, so as to get a series of optimal solutions. Then, based on these solutions, we choose the best solution according to (14) as our final optimal result.

$$
\begin{aligned}
& \operatorname{MinG}_{j}
\end{aligned}
$$

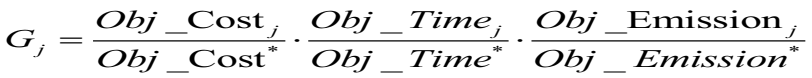

In Eq. (14), Obj_Cost ${ }_{j} . O b j \_T i m e_{j} . O b j \_$Emission ${ }_{j}$ is the corresponding objective function values ( cost、transportation time、 emission) while Obj_Cost ${ }^{*}$. Obj_Time $^{*}$ 、Obj_Emission ${ }^{*}$ is the minimal of the solutions.

On the other hand, since this model is considered to be a Location-Route problem which can be usually solved using a two-stage algorithm, i.e. firstly to solve location problem, then to search transportation routes. We use a two-stage heuristic algorithm to quickly solve the proposed model considering constraint (9), (12). The procedure is illustrated in the next section.

\subsection{Flowchart of Two-stage Heuristic Algorithm}

For the sake of clarify this two-stage heuristic algorithm, we define three problems $\mathrm{P} 0$, $\mathrm{P} 1, \mathrm{P} 2$ according to the objective functions and constraints. P0 is composed of (1)-(13). Equations (1)-(10), (12), (13) define P1 which means $x_{h}$ value is determined. P2 is composed of (1)-(8), (10), (12), (13), in which the objective function is in the second part of (1). The two-stage heuristic algorithm's procedure is described as Figure 2.

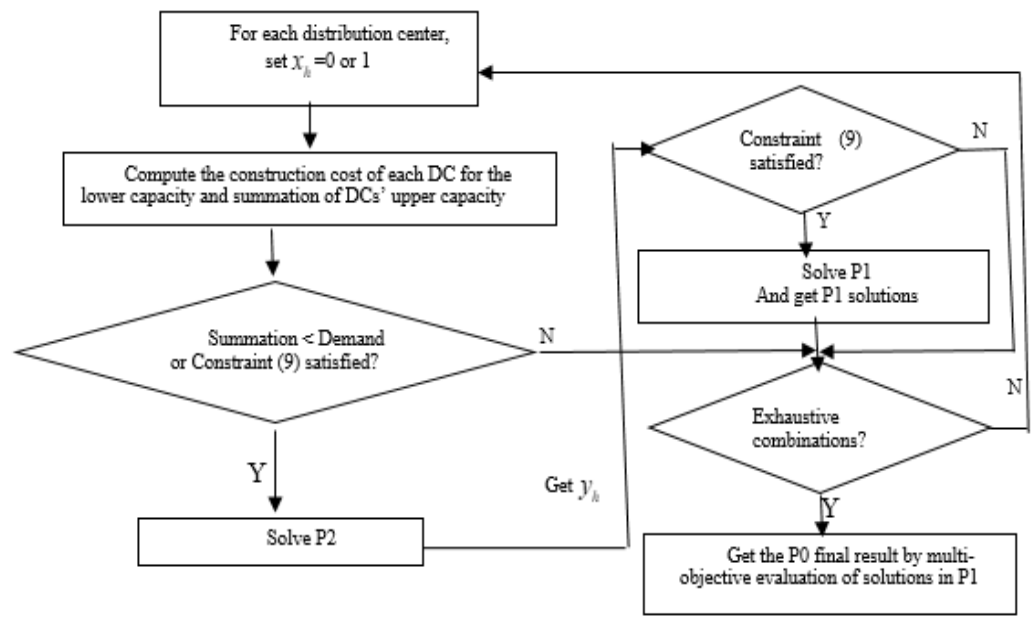

Figure 2. Flow chart of two-stage heuristic algorithm 


\section{Numerical Example Illustration}

\subsection{Parameters in Numerical Example}

To confirm the proposed model and algorithm's feasibility and effectiveness, on the basis of model and data in [9], a multi-modal transport network is designed which includes 6 products, 18 origin delivery and destination points, 8 distribution centers, 3 transport modes. Different from in [9], only three transport modes (road, rail, airline) are incorporated among origins and distribution centers and destinations. Construction cost function of distribution is listed as below: $\operatorname{In} v^{h}\left(y_{h}\right)=C_{o}^{h}+\left(y_{h}-L B_{n}\right) C_{1}^{h}$.

Parameters $C_{0}^{h}, C_{1}^{h}$, distances, supplier capacity and customer demands are produced using random functions.

There are 23328 paths in total, 139984 decision variables and 140281 constraints. Cost, time and emission of three transport modes are given in Table 3. Other parameters in the model are set as following: unit storage cost is 2.641 , unit emission is 0.6826 , storage time in distribution center is 12 , scope economy coefficient is 0.25 , total budget is 8600000 , is 10 , and the lower, upper capacity limit is 500,8000 .

Table 3. Transportation cost, time and emission for different transport mods

\begin{tabular}{clcc}
\hline $\begin{array}{l}\text { Transport mode } \\
\text { parameter function }\end{array}$ & road & rail & airline \\
\hline $\begin{array}{c}\text { Unit } \\
\text { transportation cost }\end{array}$ & $0.2782 d^{0.817}$ & $0.8705 d^{0.627}$ & $869.32 d^{0.139}$ \\
$\begin{array}{c}\text { Transportation } \\
\text { time }\end{array}$ & $0.0067 d^{1.1465}+$ & $10^{-10} \times d^{3}-10^{-5} \times d^{2}$ & $0.0023 d+$ \\
+ Unit emission & 2.823 & $0.0829 \times d+22.914$ & 19.254 \\
& $0.484 d$ & $0.0392 d$ & $1.67 d$ \\
\hline
\end{tabular}

\subsection{Results Comparison Analysis}

Based on the algorithm above designed and parameters in the previous section, the proposed multi-objective optimization model is solved in the context of CPlex solver, GAMS 24.2, RAM 4G, and CPU 1.4G. To illustrate the feasibility and effectiveness, we compare the efficiency of two-stage heuristic algorithm in Table 4 by relaxing two criterions. The first criterion is: total investment of distribution center selected with lower capacity is less than total budget or sum of upper capacity of distribution center selected is more than products demand in destination points. The second criterion is: construction cost of distribution center which is got by solving P2 is less than total budget.

Table 4. Solving efficiency based on heuristic algorithm

\begin{tabular}{ccc}
\hline Criterion 1 & Criterion 2 & Solving time \\
\hline No & No & $>120$ hours \\
No & Yes & $>60$ hours \\
Yes & No & $>20$ hours \\
Yes & Yes & $<1$ hours \\
\hline
\end{tabular}


From Table 4, it can be seen that computation efficiency is improved a lot by introducing two criterions. Actually, the execute time is about half an hour. The final evaluation results are illustrated in Table 5 which is got by evaluating 56 feasible solutions based on formula (14) (not listed here in a detailed description as space is limited).

As can be seen in Table $5,13^{\text {th }}$ solution is the best one, which suggests that distribution 1,3,4,5 should be selected, and their capacity is $8000,8000,4035$ and 6528 respectively. Compared to the maximal cost, time and emission, the percentage of cost reduction, time saving is $6.45,8.43$, while the percentage of cost, time increase and emission reduction is $1.66,41.27,41.42$ respectively. This means this optimal solution makes a better compromise among three objectives. Also, it finds there are about $92 \%$ solutions including railway transport have been suggested which implies that railway maybe an indispensable role in multi-modal transport network.

Table 5. Evaluation of multi-objective optimization solutions

\begin{tabular}{llllllll}
\hline Solution & $\begin{array}{l}\text { Index } \\
\text { Value }\end{array}$ & Solution & $\begin{array}{l}\text { Index } \\
\text { Value }\end{array}$ & Solution & $\begin{array}{l}\text { Index } \\
\text { Value }\end{array}$ & Solution & $\begin{array}{l}\text { Index } \\
\text { Value }\end{array}$ \\
\hline 1 & 1.5415 & 15 & 1.6059 & 29 & 1.7862 & 43 & 1.6920 \\
2 & 1.5846 & 16 & 1.6714 & 30 & 1.7494 & 44 & 1.6700 \\
3 & 1.5816 & 17 & 1.5786 & 31 & 1.7494 & 45 & 1.6092 \\
4 & 1.6897 & 18 & 1.6553 & 32 & 1.7761 & 46 & 1.8349 \\
5 & 1.6005 & 19 & 1.6786 & 33 & 1.6883 & 47 & 1.9377 \\
6 & 1.6213 & 20 & 1.5982 & 34 & 1.6049 & 48 & 1.7146 \\
7 & 1.7395 & 21 & 1.6739 & 35 & 1.6816 & 49 & 1.8890 \\
8 & 1.6550 & 22 & 1.7629 & 36 & 1.7468 & 50 & 1.8956 \\
9 & 1.7499 & 23 & 1.5917 & 37 & 1.7583 & 51 & 1.8401 \\
10 & 1.7128 & 24 & 1.7444 & 38 & 1.7118 & 52 & 1.8596 \\
11 & 1.7343 & 25 & 1.7761 & 39 & 1.8021 & 53 & 2.0912 \\
12 & 1.7263 & 26 & 1.6801 & 40 & 1.8592 & 54 & 2.0880 \\
13 & 1.4361 & 27 & 1.8099 & 41 & 1.7160 & 55 & 1.9473 \\
14 & 1.4702 & 28 & 1.9001 & 42 & 1.8646 & 56 & 1.9241 \\
\hline & & & & & & & \\
\hline
\end{tabular}

\section{Conclusion}

With rapid development of social economy, logistic distribution is playing a more and more important role in linking production and consumption. Transportation is considered to be a critical activity in logistics and have various positive and negative influences such as delivery, exhaust emission etc. In this paper, we propose an extended multi-objective optimization model on logistics location and route in multimodal context which is to minimize total operation cost, transportation time and emission. Then, a two-stage heuristic algorithm is designed according to the model's structure. Finally, a large scale numerical example is introduced to illustrate the model's feasibility and the algorithm's effectiveness, also it finds railway can play a critical part in multi-modal transport network. Future studies can be improved by 
introducing new elements such as inverse logistics or designing more effective algorithms.

\section{References}

[1] Sadjady H., Davoudpour H. Two-echelon, multi-commodity supply chain network design with mode selection, lead-times and inventory costs. Computers \& Operations Research, 2011, 39(7):1345-1354.

[2] Swedish Environmental Protection Agency. Environmental impact from different modes of transport. Stockholm: 2001, 4.

[3] Paksoy T, Özceylan E, Weber GW. A Multi objective model for optimization of a green supply chain network. Global Journal of Technology\& Optimization, 2011,(2):85-96.

[4] China Baike. Transportation costs' components and influencing factors [EB/OL]. http://www.chinabaike.com/ zixun/hangyezonghe/2014/1115/2996710.html, 2020-8-1.

[5] Bauer J, Bektas T, Crainic TG. Minimizing greenhouse gas emissions in intermodal freight transport: an application to rail service design. Journal of the Operational Research Society, 2010, 61(3): 530-542.

[6] Beresford A, Pettit S, Liu YK. Multimodal supply chains: iron ore from Australia to China. Supply Chain Management: An International Journal, 2011,16(1):32-42.

[7] Cho JH, Kim HS, Choi HR. An intermodal transport network planning algorithm using dynamic programming-A case study: from Busan to Rotterdam in intermodal freight routing. Appl Intell, 2012, 36:529-541.

[8] Bhattacharya A, Kumar SA, et al. An intermodal freight transport system for optimal supply chain logistics. Transportation Research Part C, 2014, 38:73-84.

[9] Bortolini M, Faccio M, Ferrari E, et al. Fresh food sustainable distribution: cost, delivery time and carbon footprint three-objective optimization. Journal of Food Engineering, 2016, 174:56-67.

[10] Hao Y, Xue Q, Cao Z, and Zhang X. A two-stage chance constrained approach with application to stochastic intermodal service network design problems. Journal of Advanced Transportation, vol. 2018:18 pages

[11] Jiang JH, Zhang DZ, Li SY, and Liu YJ. Multimodal green logistics network design of urban agglomeration with stochastic demand. Journal of Advanced Transportation, Volume 2019: 19 pages. 